\title{
Near-Wall Motion of Caged Fluorescent Dye in Microchannel Flows Obtained from Evanescent Wave Molecular Tagging*
}

\author{
Yuriko SENGA $^{* *}$, Tsubasa NAKAMURA ${ }^{* *}$, Hiroki FUKUMURA ${ }^{* *}$, \\ Mitsuhisa ICHIYANAGI ${ }^{* * *}$ and Yohei SATO** \\ **Department of System Design Engineering, Keio University, \\ 3-14-1 Hiyoshi, Kohoku-ku, Yokohama, 223-8522 \\ E-mail: yohei@sd.keio.ac.jp \\ ***Department of Mechanical Engineering, Intelligent Modeling Laboratory, \\ The University of Tokyo, \\ 7-3-1 Hongo, Bunkyo-ku, Tokyo, 113-8656
}

\begin{abstract}
A molecular tagging technique utilizing evanescent wave illumination was developed to investigate the motion of a caged fluorescent dye in the vicinity of the microchannel wall surface in electroosmotic and pressure-driven flows. A line pattern in a buffer solution was written by a pulsed UV laser and the uncaged dye was excited by the evanescent wave with total internal reflection inside the glass wall using an objective lens. The velocities calculated by the measured displacement of the near-wall tagged region were compared with the results of molecular tagging using volume illumination, which represents the bulk flow information. Concerning electroosmotic flow, the micro-PIV technique using a confocal microscope system was applied to the microchannel rinsed by the caged fluorescein beforehand in comparison with a pure glass-PDMS microchannel to examine the effect of dye adsorption to the wall on the electroosmotic mobility. The electroosmotic mobility obtained by evanescent wave molecular tagging (EWMT) showed close to the micro-PIV measurement result near the glass wall for the rinsed case and the uncaged dye at the almost constant velocity remained in the depthwise illumination region. On the other hand, the dye velocity in pressure-driven flow by EWMT increased rapidly with respect to time. The uncaged dye convected to the streamwise direction dispersed toward the wall due to the concentration gradient of the dye, which was confirmed by the numerical simulations.
\end{abstract}

Key words: Microchannel, Caged Fluorescent Dye, Dye Adsorption, Evanescent Wave, Molecular Tagging

\section{Introduction}

The precise control of a small liquid volume and molecules in microchannel flows is expected to improve the efficiency of analysis and detection in micro total analysis systems $^{(1),(2)}$, which are dependent on the development of accurate measurement techniques. The optical methodology to understand the fluid motion in microchannels falls into two categories $^{(3)}$ : tracking fluorescent particles without disturbing fluid flow and tracing fluorescent dye dissolved in a solution. Micron-resolution particle image velocimetry

${ }^{*}$ Received 16 Nov., 2009 (No. 09-0695) [DOI: 10.1299/jfst.5.192]

Copyright $\odot 2010$ by JSME 
(micro-PIV) has become a fundamental tool to design the microchannel geometry for integrating the microchannels into the microfluidic device ${ }^{(4)}$. In addition, spatial averaged time-resolved particle tracking velocimetry ${ }^{(5)}$ has enabled us to obtain the velocity-vector map of fluctuating flow without losing a temporal resolution. For further insight into fluid dynamics near the liquid-solid interface, nano-PIV ${ }^{(6),(7)}$ has been developed utilizing the evanescent wave, which is generated from total internal reflection of light. In the experiments by these particle-based velocity measurements, however, it is not easy to avoid the particle agglomeration and adhesion to the wall. Thus the dye-based velocity measurement is the method of choice for understanding the motion of molecules in the vicinity of the wall.

Tagging and tracing molecules in the regions of interest, a.k.a., molecular tagging velocimetry (MTV), is an alternative optical approach to investigate the flow structure. Caged fluorescent dye is commonly used, because of its high signal-to-noise ratio and its fidelity to the fluid motion. Experimental efforts by Gendrich and Koochesfahani ${ }^{(8)}$, and Koochesfahani et al. ${ }^{(9)}$ have contributed to improve the measurement accuracy of MTV in macroscale flows and to enhance its feature. Application of MTV to microchannel flows has been conducted by Paul et al. ${ }^{(10)}$, Herr et al. ${ }^{(1)}$, Ross et al. ${ }^{(12)}$, Maynes and Webb ${ }^{(13)}$ and Sinton et al. ${ }^{(14)}$ and Sinton and $\mathrm{Li}^{(15)}$. In these works the uncaged region was illuminated by a $\mathrm{CW}$ laser or a mercury lamp (hereinafter refer to as volume illumination), thus the captured images contained the bulk flow information of microchannel. This means that it is hardly to detect the near-wall motion of molecules, even if the microchannel wall is focused with a thin depth-of-field.

At the initial stage the capability of MTV has been examined in the experiments of pressure-driven microchannel flow, because the theoretical velocity profiles at the low Reynolds number have been easily calculated ${ }^{(16)}$ and compared with the measured ones. In this type of flow the dye adsorption to the wall has no significant influence on the flow structure. However, in electrokinetically driven flow, this causes the drastic change in the electrostatic potential of the wall, i.e., the zeta-potential ${ }^{(17)}$, which directly induces an increase or a decrease in the electroosmotic flow velocity and a change in the velocity gradient in the depthwise direction. On the other hand, in the past experiments, the uncaged dye convected to the streamwise direction is of interest in spite of the dye dispersion in the depthwise direction, which has been hardly detected by MTV with volume illumination. The effect of velocity gradient on the molecule dispersion affects significantly the performance of chromatographic separations using microfluidic device ${ }^{(18)}$, however, none of the experiments has focused on the near-wall behavior. The optical diagnostic with the near-wall resolution is required to extend our understanding of the molecule adsorption effect on interfacial flow and the molecule dispersion near the wall.

The objective of the present study is to develop a molecular tagging technique in combination with evanescent wave illumination for investigation of the effect of dye adsorption to the wall on electroosmotic flow velocities, because the zeta-potential, is drastically changed by the dye. Caged fluorescent dye is selected, because the high contrast images are easily obtained just after cleaving a caging group by a pulsed UV laser. The uncaged dye existed in the vicinity of the glass wall is excited by the evanescent wave using an objective lens with a high numerical aperture. Evanescent wave molecular tagging (hereinafter refer to as EWMT) is applied to electroosmotic flow in the glass-PDMS microchannel and the measurement results are compared to those using the microchannel rinsed by the CMNB-caged fluorescein measured by micro-PIV. Moreover the dye dispersion near the wall in pressure-driven flow is measured by EWMT. The effect of velocity gradient on the dye dispersion near the wall is examined in comparison with numerical simulations. Important conclusions obtained in the present study will contribute to handle nanoscopic objects at the electrolyte-solid interface. 


\section{Nomenclature}

$D \quad$ : dye diffusivity

E : electric field

$I_{e} \quad$ : excitation light intensity

$I_{\text {eva }} \quad$ : evanescent wave intensity

$I_{f} \quad$ : fluorescent intensity from dye

$n_{1} \quad$ : refractive index of borosilicate cover glass

$n_{2} \quad$ : refractive index of buffer solution

$t \quad:$ time

$U \quad$ : streamwise velocity

$U_{\mathrm{EP}}$ : electrophoretic velocity of dye

$x, y, z$ : Cartesian coordinate system

$z_{p} \quad$ : characteristic penetration depth of the evanescent wave

$\lambda \quad:$ laser wavelength

$\mu_{\mathrm{EP}} \quad$ : electrophoretic mobility of the dye

$\theta_{i} \quad$ : incidence angle

\section{Experimental Setup}

\subsection{Measurement Principle}

Caged fluorescent dye is initially non-fluorescent in a buffer solution, because a chemical group to quench the fluorescence is attached. Just after the dye is irradiated by an ultraviolet laser pulse, the chemical group is cleaved and the dye restores the fluorescence. This uncaging event is irreversible and requires on the order of milliseconds at least ${ }^{(19)}$. In MTV the uncaged dye is excited and the fluorescence images in time series are captured by a camera to calculate the dye displacement. An epi-fluorescent microscope system has been usually employed to excite the uncaged dye using a CW laser ${ }^{(10),(15)}$ or a mercury lamp ${ }^{(12)}$, as illustrated in Figure 1(a). The fluorescence from the dye in the whole depthwise direction is illuminated, which means that the bulk flow information is obtained. The advantage of the EWMT technique is that the evanescent wave is utilized to excite the uncaged dye near the glass wall, as shown in Figure 1(b), so that the near-wall motion of dye can be captured.

The evanescent wave is generated by total internal reflection inside the glass wall and its intensity, $I_{\text {eva }}$, decays exponentially in terms of the depthwise distance, $z$, from the glass wall, as shown in Figure 1(b), which is defined by

$$
I_{\text {eva }}(z)=I_{\text {eva }}(0) \exp \left(-z / z_{p}\right),
$$

where $I_{\text {eva }}(0)$ is the evanescent wave intensity at the wall and $z_{p}$ is the characteristic penetration depth of the evanescent wave, given by

$$
z_{p}=\frac{\lambda}{4 \pi \sqrt{n_{1}^{2} \sin ^{2} \theta_{i}-n_{2}^{2}}},
$$

where $\lambda$ is the laser wavelength in a vacuum. In the present experiments, $z_{p}$ was calculated to be $76 \mathrm{~nm}$ with the refractive index of a borosilicate cover glass, $n_{1}=1.523$, and a buffer solution, $n_{2}=1.338$, the laser wavelength of $\lambda=488 \mathrm{~nm}$ and the incidence angle of $\theta_{i}=70^{\circ}$. This angle was calculated by ${ }^{(20)}$

$$
L=f_{\text {obj }} \cdot n_{\text {oil }} \sin \theta_{i},
$$

where $L$ is the distance from the center of the objective lens to the off-center location of the laser beam, $f_{\text {obj }}$ is the focal length of the objective lens and $n_{\text {oil }}$ is the refractive index of the index-matching oil of 1.515 . The evanescent wave intensity is reduced to less than $1 \%$ at $z=$ 
(a)
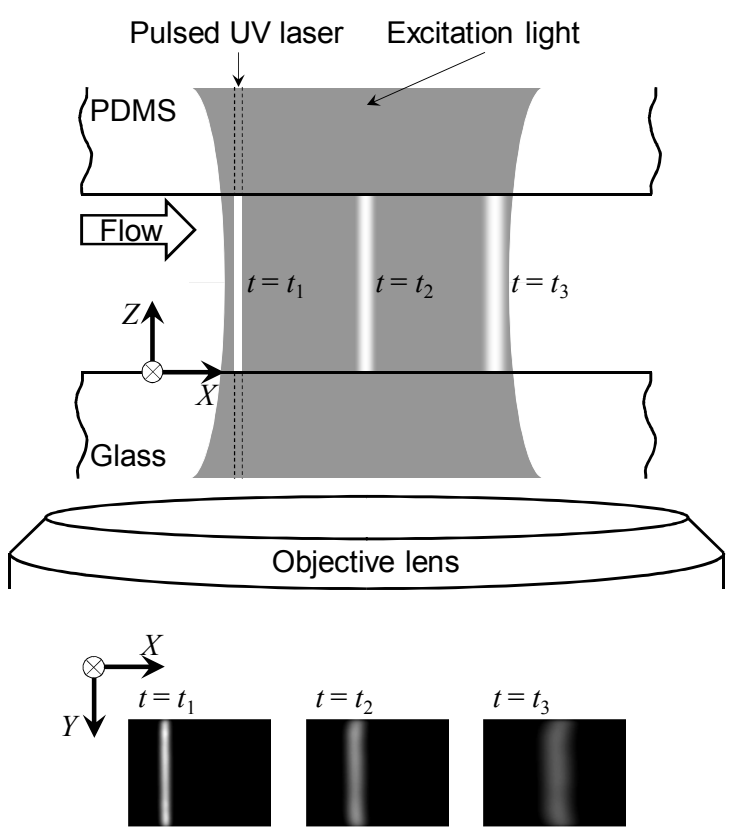

(b)

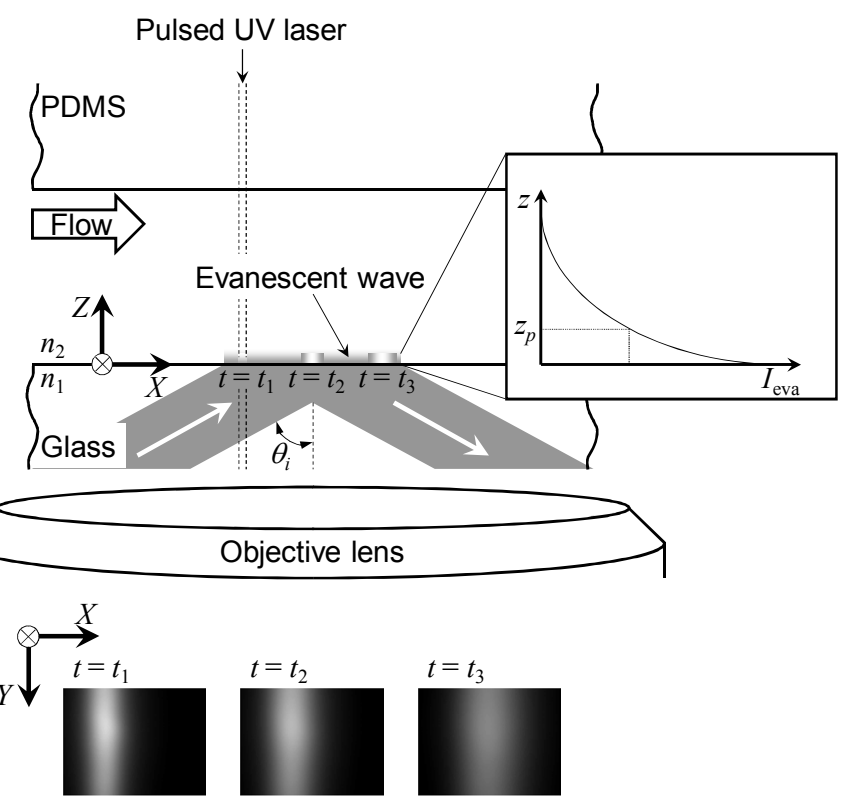

Figure 1. Schematic concept of (a) molecular tagging (MT) by volume illumination and (b) evanescent wave molecular tagging (EWMT).

$550 \mathrm{~nm}$.

\subsection{Properties of Caged Fluorescent Dye}

Paul et al. ${ }^{(10)}$ reported that the undesired separation effect in electrokinetically driven flow was observed due to the multicomponent nature of the caged fluorescent dye, e.g., 4, 5-dimethoxy-2-nitrobenzyl (DMNB)-caged fluorescein dextran. Therefore fluorescein bis-(5-carboxymethoxy-2-nitrobenzyl)ether, dipotassium salt (CMNB)-caged fluorescein, i.e., the single component dye, supplied by Invitrogen, Corp. was selected as the dye tracer, whose properties are listed in Table 1 . The CMNB-caged fluorescein of $0.5 \mathrm{mmol} / l$ was dissolved in a $10 \mathrm{mmol} / l$ carbonate buffer at a $\mathrm{pH}$ of 9.5 . The solution was filtered by 0.2 $\mu \mathrm{m}$ pore syringe filters. 
Table 1. Properties of CMNB-caged fluorescein

Chemical formula

Molecular weight

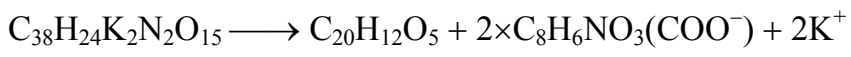
826.81

Adsorption wavelength for photolysis (nm) 353

Adsorption wavelength

(nm) $\quad 494$

Emission wavelength

(nm) $\quad 518$

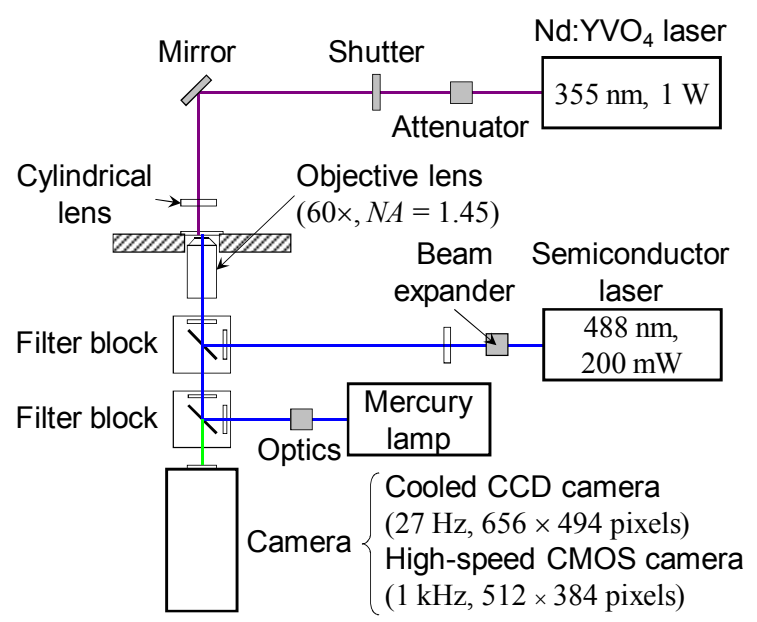

Figure 2. Schematic of the optical measurement system.

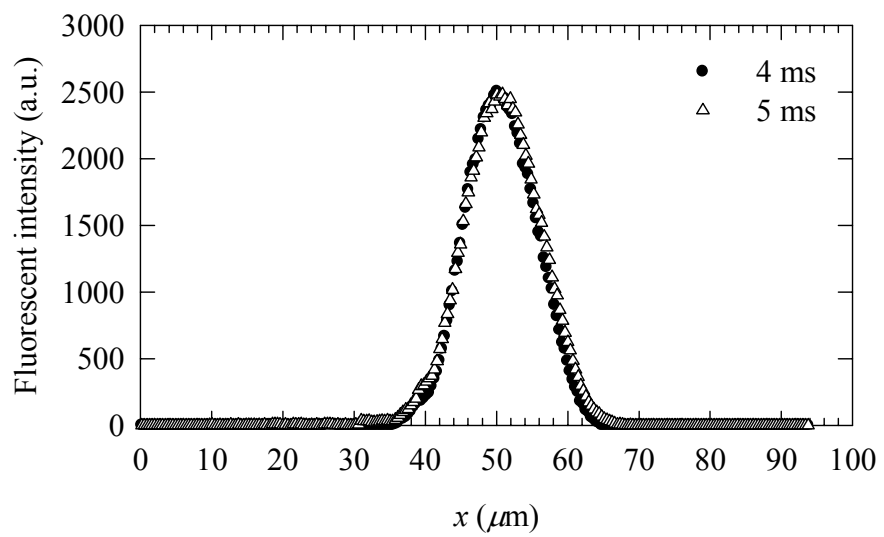

Figure 3. Fluorescent intensity profiles of the uncaging event by the pulsed UV laser. The thickness of the laser sheet was estimated to be $15 \mu \mathrm{m}$.

\subsection{Optical Setup}

The optical setup in the present study was established for the both uses of EWMT and molecular tagging (hereinafter refer to as MT) by volume illumination. Figure 2 exhibits a schematic of the measurement system based on an inverted microscope (Nikon Corp., TE2000U). Two types of camera were employed in consideration of the maximum flow velocity: (1) a cooled CCD camera (Hamamatsu Photonics K.K., C4880-80, $656 \times 494$ pixels, 12 bits, $27 \mathrm{~Hz}$ ) for measurements of the dye diffusivity in stationary flow and the maximum velocity of $100 \mu \mathrm{m} / \mathrm{s}$, (2) a high-speed CMOS camera (Vision Research, Inc., Phantom V7.1, $512 \times 384$ pixels, 12 bits, $1 \mathrm{kHz}$ ) for the maximum velocity of $3 \mathrm{~mm} / \mathrm{s}$. An $\mathrm{Nd}: \mathrm{YVO}_{4}$ laser (DPSS, Inc., 3510-30) operating at a wavelength of $355 \mathrm{~nm}$ and a power of $1 \mathrm{~W}$ was used to cleave the caging group. The laser beam whose power was controlled to be $0.1 \mathrm{~W}$ by an attenuator was pulsed by a mechanical shutter (Vincent Associates Inc., VMM-T1) in a pulse duration of $3 \mathrm{~ms}(t=0-3 \mathrm{~ms})$. The pulse beam was focused by a 


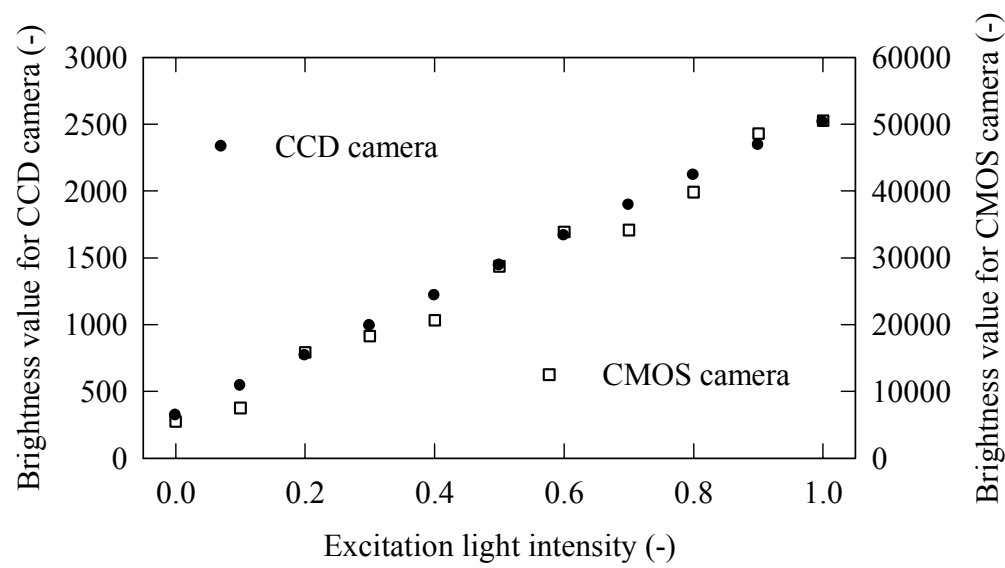

Figure 4. Sensor linearity of CCD and CMOS cameras. Detected brightness values were plotted against excitation light intensity.

plano-convex cylindrical lens of $f=50 \mathrm{~mm}$, which produced a laser sheet with a thickness of approximately $15 \mu \mathrm{m}$. Figure 3 shows fluorescent intensity profiles in stationary flow captured by the high-speed CMOS camera. After the profile at $t=4 \mathrm{~ms}$ (solid circle marks) was fitted with a Gaussian function, the laser sheet thickness was estimated by $1 / e$ value of the fluorescent intensity.

The volume illumination was provided by a continuous mercury lamp, whose light was routed through a bandpass filter (450-490 nm) and a dichroic mirror transmitting wavelengths above $505 \mathrm{~nm}$ onto an I-shaped microchannel. On the other hand, the evanescent wave illumination system was constructed based on a $60 \times$ magnification, oil-immersion objective lens (Nikon Corp., CFI Plan Apo TIRF) with a numerical aperture of 1.45. A laser beam from an optically pumped semiconductor laser (Coherent, Inc., Sapphire 488-200) operating at a wavelength of $488 \mathrm{~nm}$ and a power of $200 \mathrm{~mW}$ was expanded. The expanded beam was focused on the back focal plane at the peripherals region of the objective lens after passing through a plano-convex lens of $f=600 \mathrm{~mm}$ and a dichroic mirror transmitting wavelengths above $505 \mathrm{~nm}$. The collimated laser beam from the objective lens was introduced into an immersion oil with the refractive index of 1.515 and finally total internal reflection was occurred at the interface between the borosilicate cover glass and the buffer solution. The total internal reflection angle was adjusted to be $70^{\circ}$, which yielded the characteristic penetration depth of $76 \mathrm{~nm}$.

The fluorescence images were collected onto the pixel sheet of the camera through the objective lens, an emission filter transmitting wavelengths longer than $520 \mathrm{~nm}$ and a $0.6 \times$ magnification TV lens. The size of the CCD pixel sheet along with the $60 \times$ objective lens corresponded to $180 \mu \mathrm{m} \times 136 \mu \mathrm{m}$, while the size of the CMOS pixel sheet corresponded to $188 \mu \mathrm{m} \times 141 \mu \mathrm{m}$. In the present study, the main factor of the difference between both cameras in affecting the measurement accuracy is the sensor linearity ${ }^{(21)}$. Figure 4 shows the brightness value detected by both cameras in terms of the excitation light intensity. A non-linear effect was not observed in the CCD camera, while the slight effect can be found in the CMOS camera. Due to the limitation of frame rate of CCD camera, the CMOS camera was applied to the maximum velocity of $3 \mathrm{~mm} / \mathrm{s}$.

\subsection{Image Processing and Data Acquisition}

An average per $5 \times 5$ pixels was applied to all captured images for reducing the pixel errors. This averaging technique, the pixel size, and the magnification of the objective lens and the TV lens determined a spatial resolution of the molecular tagging technique. The spatial resolution resulted in $1.4 \mu \mathrm{m} \times 1.4 \mu \mathrm{m}$ using the CCD camera, while $1.85 \mu \mathrm{m} \times 1.85$ 
(a)

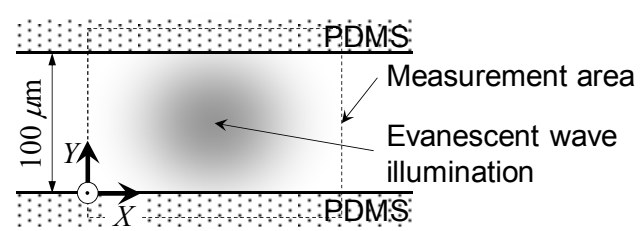

(c)

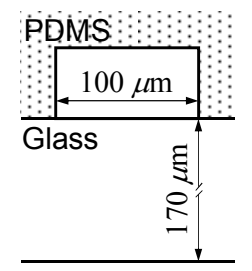

(b)

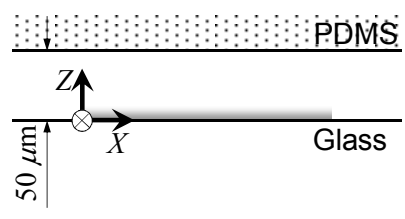

(d)

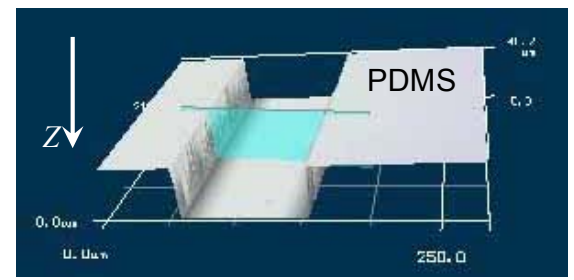

Figure 5. Schematic of $(a)$ top, $(b)$ side and $(c)$ cross-sectional views of the I-shaped microchannel. $(d)$ The width and depth of the PDMS microchannel were measured by a 3D laser scanning microscope.

using the CMOS camera. Subsequent post-processing was carried out to remove any non-uniformities of the excitation light intensity from the light source and of the background noise. The following normalization was applied to all captured images ${ }^{(15),(22)}$

$$
I_{n}=\frac{I_{f}-I_{b 1}}{I_{e}-I_{b 2}},
$$

where $I_{n}$ is the normalized fluorescent intensity, $I_{f}$ is the fluorescent intensity from the dye and $I_{e}$ is the excitation light intensity (or the bright field image). Prior to each run, $I_{e}$ was captured at an exposure time of $36 \mathrm{~ms}$ of the CCD camera (or $940 \mu \mathrm{s}$ for the CMOS camera), when the uncaged dye in the buffer solution was illuminated by the evanescent wave or the mercury lamp. The background noise, $I_{b 2}$, was obtained by a captured dark-field image at an exposure time of $36 \mathrm{~ms}$ and subtracted from $I_{e}$. Then the background noise, $I_{b 1}$, was obtained by a captured dark-field image right before each measurement and subtracted from $I_{f}$. The exposure times for $I_{b 1}$ and $I_{f}$ were set to be a same value and dependent on each measurement.

\subsection{Microchannel flow system}

Figure 5 illustrates an I-shaped microchannel comprised of a poly(dimethysiloxane) (PDMS) chip fabricated by a replica moulding technique and a borosilicate cover glass with a thickness of $170 \mu \mathrm{m}$. Measurements of the width and the depth by a 3D laser scanning microscope (Keyence Corp., VK-9700) showed $100 \mu \mathrm{m}$ and $50 \mu \mathrm{m}$, respectively. Cylindrical reservoirs were attached to the inlet and the outlet. Electroosmotic flow was generated by applying a DC electric field along the microchannel between platinum electrodes submerged into the reservoirs. The origin of the Cartesian coordinate system was located at the bottom left corner of the measurement area, as illustrated in Figure 5.

\section{Results and Discussion}

\subsection{Diffusivity and Electrophoretic Mobility of Dye}

The dye velocity in electroosmotic flow represents the summation of the electroosmotic velocity of the buffer solution and the electrophoretic velocity of the dye ${ }^{(14)}$. Firstly the dye diffusivity in stationary flow of the I-shaped microchannel was measured by EWMT and 
Table 2. Diffusivity and electrophoretic mobility of CMNB-caged fluorescein

\begin{tabular}{llc}
\hline & $\begin{array}{c}\text { Diffusivity } \\
\left(\times 10^{-10} \mathrm{~m}^{2} / \mathrm{s}\right)\end{array}$ & $\begin{array}{c}\text { Electrophoretic mobility } \\
\left(\times 10^{-8} \mathrm{~m}^{2} /(\mathrm{V} \cdot \mathrm{s})\right)\end{array}$ \\
Evanescent wave molecular tagging & $5.05 \pm 0.42$ & $-3.89 \pm 0.32$ \\
Molecular tagging by volume illumination & $5.46 \pm 0.23$ & $-4.21 \pm 0.18$ \\
Galambos and Forster ${ }^{(24)}+$ & $6.4-6.6$ & \\
Paul et al. ${ }^{(10)}+$ & 4.37 & -3.3 \\
Sinton and $\mathrm{Li}^{(15)}+$ & & -3.3 \\
\hline
\end{tabular}

+ Diffusivity of fluorescein

MT with volume illumination using the CCD camera at a frame interval of $37 \mathrm{~ms}$. The UV pulse beam was focused in the center of the measurement area. Secondly the electrophoretic mobility of the dye, i.e., the electroophoretic velocity divided by the applied electric field, was calculated by the Nernst-Einstein equation ${ }^{(23)}$. The dye diffusivity, $D$, was calculated by

$$
D=\frac{\sigma_{t=t_{i+1}}^{2}-\sigma_{t=t_{i}}^{2}}{2 \Delta t},
$$

where $\Delta t$ is the frame interval of the CCD camera, $\sigma_{t=t_{i}}^{2}$ and $\sigma_{t=t_{i+1}}^{2}$ are the variances of the fluorescent intensity profile at $t=t_{i}$ and $t_{i+1}$, respectively, which were calculated by fitting the profiles with a Gaussian function ${ }^{(18)}$. This operation was applied to the captured images just after $74 \mathrm{~ms}$ of the UV laser exposure. The diffusivity of the CMNB-caged fluorescein is compiled in Table 2 in comparison with the experimental results by Galambos and Forster $^{(24)}$ and Paul et al. ${ }^{(10)}$. The dye diffusivity by Galambos and Forster ${ }^{(24)}$ shows larger than that by both EWMT and MT, because they conducted their experiments using fluorescein dissolved in a pure water. The uncertainties were calculated using the one-standard-deviation statistical uncertainties in each set of measurements. The electrophoretic mobility of the dye, $\mu_{\mathrm{EP}}$, was calculated by the Nernst-Einstein equation

$$
\mu_{\mathrm{EP}}=\frac{U_{\mathrm{EP}}}{E}=\frac{z N_{A} e D}{R T},
$$

where $U_{\mathrm{EP}}$ is the electrophoretic velocity of dye, $E$ is the electric field, $z$ is the valence of dye, $N_{A}$ is the Avogadro Constant, $e$ is the elementary charge, $R$ is the gas constant and $T$ is the temperature of solution. The electrophoretic mobility by EWMT show reasonable agreement with the reports by Paul et al. ${ }^{(10)}$ and Sinton and $\mathrm{Li}^{(15)}$. The difference between their values and the present data is caused by the different dye concentration and $\mathrm{pH}$ of buffer solution. Paul et al. ${ }^{(10)}$ utilized a $0.1 \mathrm{mM}$ DMNB-caged fluorescein dextran and a carbonate buffer at a $\mathrm{pH}$ of 8.0 and Sinton and $\mathrm{Li}^{(15)}$ used a $1 \mathrm{mM}$ CMNB-caged fluorescein and a carbonate buffer at a $\mathrm{pH}$ of 8.0.

\subsection{Effect of Dye Adsorption to Wall on Electroosmotic Flow}

The instantaneous velocity of the dye was determined by the displacement between the brightest intensity of two successive images divided by the frame interval of the camera. Figure 6 shows one of examples of fluorescent intensity profiles by EWMT using the CMOS camera. The velocity was obtained using the fluorescent intensity profiles after 74 $\mathrm{ms}$ of the UV laser exposure in all the sets of experiments, thus, for example, $4 \mathrm{~ms}$ in Figure 6 corresponds to $t=t_{0}+4 \mathrm{~ms}\left(t_{0}=74 \mathrm{~ms}\right)$. When these profiles were fitted with a Gaussian function, the measurement error was approximately $70 \%$ against the instantaneous velocity calculated using the peak values of the original profiles. Thus in the present study, the displacement was calculated using the peak values.

The height of water surface in the two reservoirs was carefully balanced to avoid pressure-driven flow. Elelctroosmotic flows on application of $333 \mathrm{~V} / \mathrm{cm}$ and $37 \mathrm{~V} / \mathrm{cm}$ were measured by the CMOS and CCD cameras, respectively. Subtracting the electrophoretic 


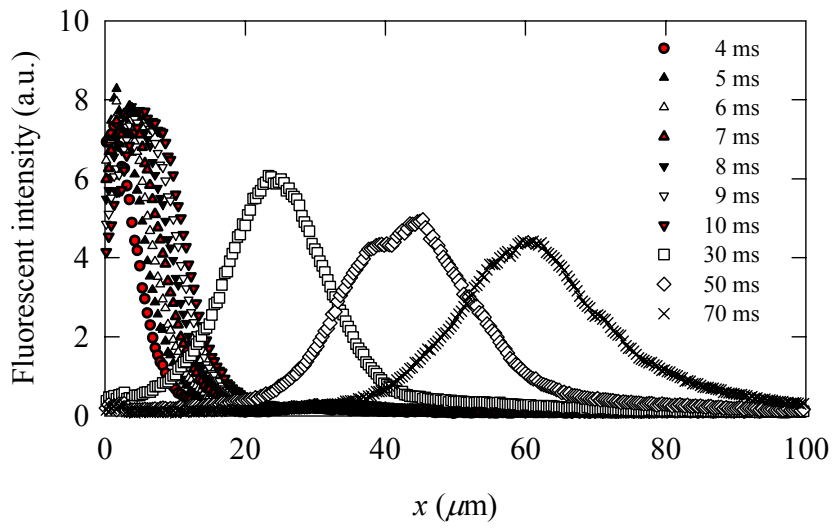

Figure 6. Fluorescent intensity profiles of electroosmotic flow in time series at $y=50 \mu \mathrm{m}$ obtained from EWMT using the CMOS camera. An electric field of $333 \mathrm{~V} / \mathrm{cm}$ was applied. Measurement was performed just after $74 \mathrm{~ms}$ of the UV laser exposure, thus, for example, 4 ms corresponds to $t=t_{0}+4 \mathrm{~ms}\left(t_{0}=74 \mathrm{~ms}\right)$.

(a)

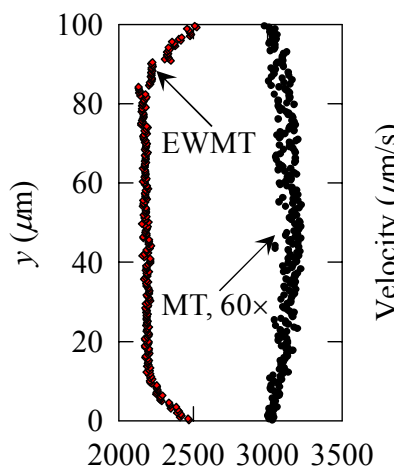

Velocity $(\mu \mathrm{m} / \mathrm{s})$ (b)

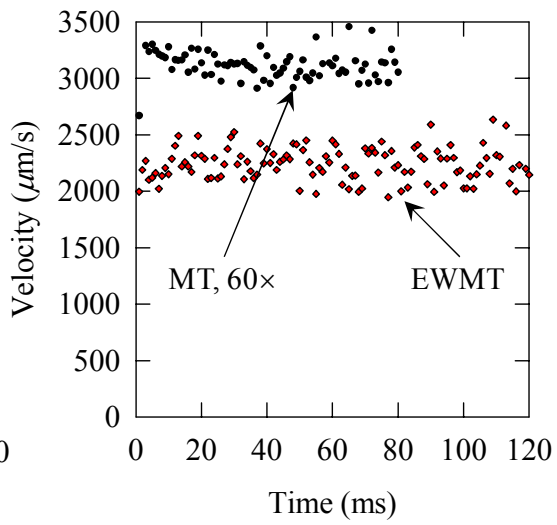

(c)

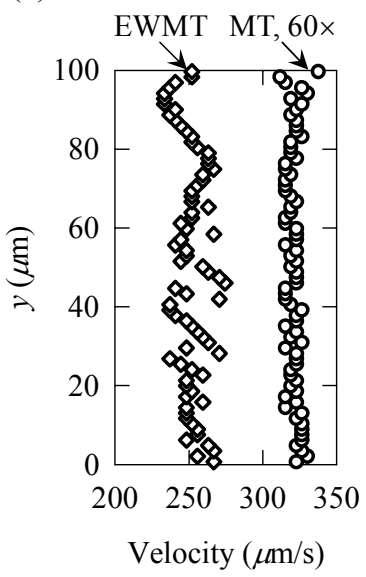

Figure 7. (a) Velocity profiles of electroosmotic flow and $(b)$ the time evolution of the electroosmotic flow velocity at $y=50 \mu \mathrm{m}$ obtained from EWMT and MT by volume illumination with the $60 \times$ objective lens using the CMOS camera. An electric field of 333 $\mathrm{V} / \mathrm{cm}$ was applied. Measurement was performed just after $74 \mathrm{~ms}$ of the UV laser exposure, thus, for example, $4 \mathrm{~ms}$ corresponds to $t=t_{0}+4 \mathrm{~ms}\left(t_{0}=74 \mathrm{~ms}\right)$. (c) Velocity profiles of electroosmotic flow obtained from EWMT and MT by volume illumination with the 60× objective lens using the CCD camera. An electric field of $37 \mathrm{~V} / \mathrm{cm}$ was applied.

velocity of the dye calculated by equation (6) from the measured dye velocity yielded the electroosmotic flow velocity. The velocity profiles using the CMOS and CCD cameras were plotted in Figures $7(a)$ and $7(c)$, respectively, and the time evolution of the velocity at $y=$ $50 \mu \mathrm{m}$ by the CMOS camera is shown in Figure $7(b)$. In MT by volume illumination, the midpoint in the depthwise (Z-) direction was focused with a depth-of-field of $0.76 \mu \mathrm{m}$ using the CMOS camera (or $0.66 \mu \mathrm{m}$ using the CCD camera). The ensemble averaging was performed using 120 images for EWMT in Figure 7(a), 80 for MT in Figure 7(a), 29 for EWMT in Figure 7(c) and 17 for MT in Figure 7(c). It is noted from Figures 7(a) and 7(c) that the velocities by EWMT are smaller than those by MT. The velocities by EWMT apparently indicate the EOF velocities near the glass wall, while those by MT may show the velocities at around the middle region, as illustrated in Figure 8. This means that that the velocity gradient in the depthwise $(Z)$ direction may be formed in the glass-PDMS microchannel. 


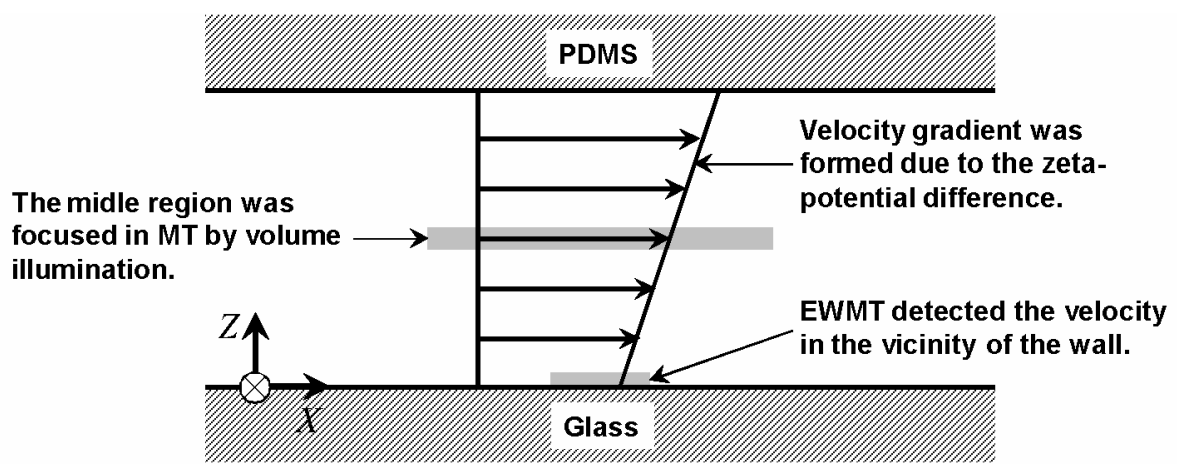

Figure 8. Schematic concept of EOF velocity distribution in the depthwise direction in the microchannel rinsed by the CMNB-caged fluorescein.

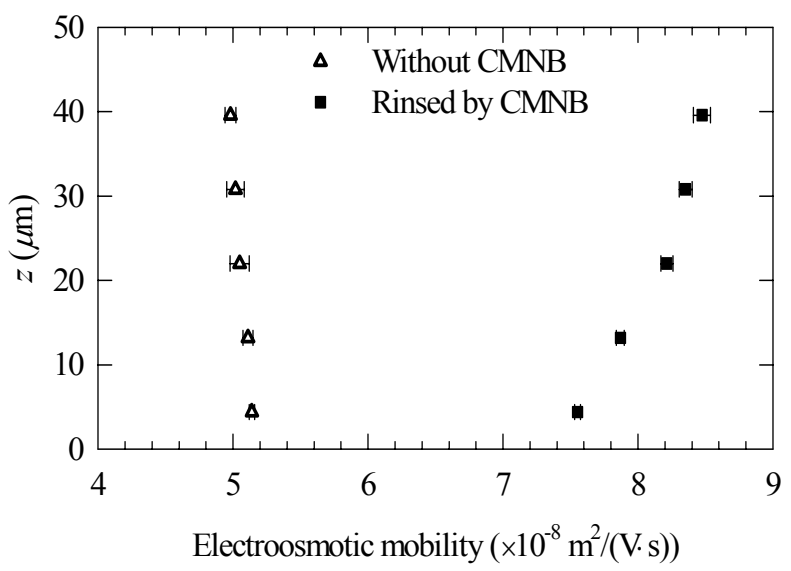

Figure 9. Electroosmotic mobility profiles in the depthwise (Z-) direction at $y=50 \mu \mathrm{m} . z=$ 0 corresponds to the glass wall, while $z=50 \mu \mathrm{m}$ corresponds to the PDMS wall. The micro-PIV technique using the confocal scanner was applied to the glass-PDMS microchannel without and rinsed by the CMNB-caged fluorescein. An electric field of 37 V/cm was applied.

The validation of velocity gradient was performed by the micro-PIV measurements of elelctroosmotic flow using a microchannel rinsed by a carbonate buffer including the CMNB-caged fluorescein beforehand (hereinafter refer to as a microchannel rinsed by the CMNB-caged fluorescein) in comparison with using a pure glass-PDMS microchannel (hereinafter refer to as a microchannel without the CMNB-caged fluorescein). The reason why the microchannel rinsed by the CMNB-caged fluorescein was prepared is that the dye adsorption to the wall affects significantly the wall zeta-potential ${ }^{(17)}$, which changes the EOF velocity and the velocity gradient in the depthwise direction. Fluorescent particles with a diameter of $500 \mathrm{~nm}$ (Invitrogen, Inc., absorption wavelength of $505 \mathrm{~nm}$, emission wavelength of $515 \mathrm{~nm}$ ) at a volumetric concentration of $0.01 \%$ was injected into a 10 $\mathrm{mmol} / \mathrm{l}$ carbonate buffer at a $\mathrm{pH}$ of 9.5 . Prior to the measurements of electroosmotic flow, the electrophoretic velocity of the particles was measured by the current monitoring technique ${ }^{(5),}$ (25). The electroosmotic flow velocity was calculated by subtracting the electrophoretic velocity from the measured velocity and the ensemble averaging was carried out using 100 velocity-vector maps. A confocal scanner (Yokogawa Electric Corp., CSU22 $\beta$ ) was applied to obtain a depth resolution ${ }^{(26),(27)}$ was $1.42 \mu \mathrm{m}$ and a spatial resolution of $27.5 \mu \mathrm{m} \times 27.5 \mu \mathrm{m}$ based upon the size of the first interrogation window.

Figure 9 shows electroosmotic mobility profiles in the depthwise (Z-) direction at $y=$ $50 \mu \mathrm{m}$ for the glass-PDMS microchannel without and rinsed by the CMNB-caged 
(a)

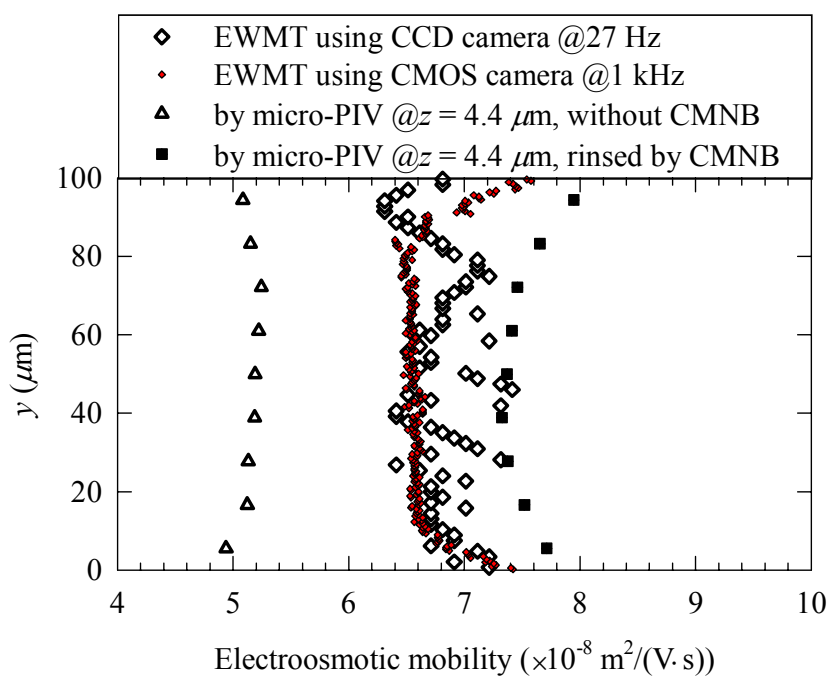

(b)

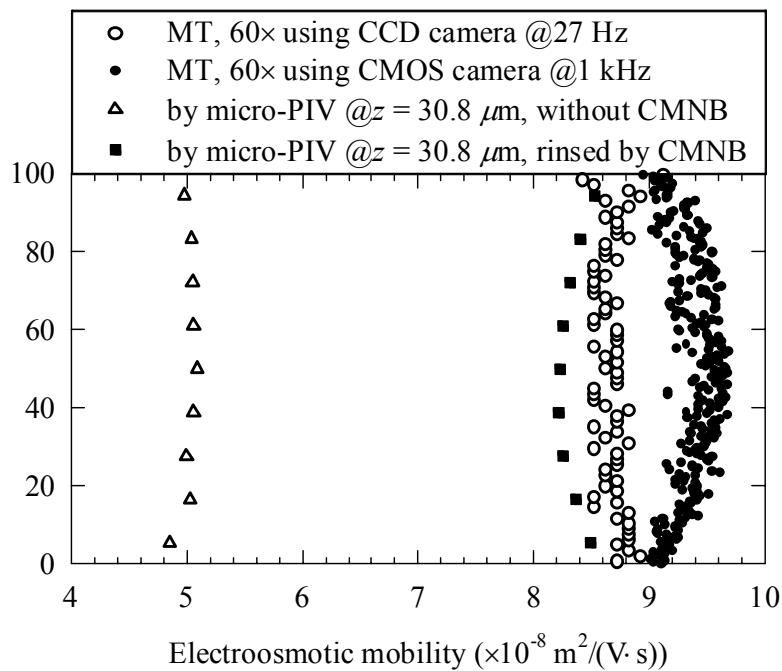

Figure 10. Electroosmotic mobility profiles in the transverse $(Y-)$ direction obtained from (a) EWMT and (b) MT by volume illumination with the $60 \times$ objective lens in comparison with the measurement results by micro-PIV.

fluorescein. The electroosmotic mobility was calculated by the elelctoosmotic flow velocity divided by an electric field of $37 \mathrm{~V} / \mathrm{cm}$. In the microchannel without the CMNB-caged fluorescein, the electroosmotic mobilities of the borosilicate glass and PDMS walls are estimated at $5.16 \times 10^{-8} \mathrm{~m}^{2} /(\mathrm{V} \cdot \mathrm{s})$ (this value gives the zeta-potential of $-66.2 \mathrm{mV}$ ) and $4.93 \times 10^{-8} \mathrm{~m}^{2} /(\mathrm{V} \cdot \mathrm{s})(-63.3 \mathrm{mV})$, respectively, which are in reasonable agreement with the values of $5.6 \times 10^{-8} \mathrm{~m}^{2} /(\mathrm{V} \cdot \mathrm{s})$ and $4.3 \times 10^{-8} \mathrm{~m}^{2} /(\mathrm{V} \cdot \mathrm{s})$ reported by Ross and Locascio ${ }^{(17)}$. On the other hand, for the microchannel rinsed by the CMNB-caged fluorescein, the electroosmotic mobility of the PDMS wall shows larger, so that the velocity gradient was formed in the depthwise (Z-) direction. According to the reports by Ross and Locascio ${ }^{(17)}$, the electroosmotic mobility (or the zeta-potential) of the glass wall was smaller than that of the PDMS wall, when the both walls were rinsed by a solution including the caged fluorescent dye. An understanding deduced from the results by the present study and Ross and Locascio $^{(17)}$ is that EWMT can capture the velocity field near the glass wall, while MT by volume illumination may capture the velocity field affected by the zeta-potential of the PDMS wall. 
The electroosmotic mobilities in the transverse $(Y-)$ direction obtained from EWMT and MT by volume illumination were plotted in Figure 10 in comparison with the results by micro-PIV. The measurement results by EWMT indicate close to the profile at $z=4.4 \mu \mathrm{m}$ for the rinsed case, as shown in Figure 10(a). To neglect the effect of particle adhesion to the glass wall rinsed by the CMNB-caged fluorescein, the near-wall velocity measurement by micro-PIV was performed at $z=4.4 \mu \mathrm{m}$. In fact the focused depthwise position by EWMT $(z \approx 175 \mathrm{~nm}$ ) was quite different from that by micro-PIV $(z=4.4 \mu \mathrm{m})$. Thus the wall velocity was estimated by extrapolating the velocity data in Figures 9 and 10. The electroosmotic mobility of the borosilicate glass wall rinsed by the CMNB-caged fluorescein at $y=50 \mu \mathrm{m}$ by micro-PIV is estimated at $7.0 \times 10^{-8} \mathrm{~m}^{2} /(\mathrm{V} \cdot \mathrm{s})$ (the zeta-potential of $-89.7 \mathrm{mV}$ ), while EWMT gave $6.6-7.0 \times 10^{-8} \mathrm{~m}^{2} /(\mathrm{V} \cdot \mathrm{s})$, thus EWMT has an ability to measure the velocity field near the wall afffected by the change in the zeta-potential due to the dye adsorption to the glass wall. The thickness of the electric double layer ${ }^{(23)}$ is calculated to be $3 \mathrm{~nm}$ in the present experimental condition. This means that the depthwise illumination region by EWMT is estimated far beyond the electric double layer. On the other hand, the results by MT using both the cameras show close to the profile at $z=30.8$ $\mu \mathrm{m}$ in the rinsed microchannel rather than the pure microchannel, as shown in Figure $10(b)$. It is noted from Figure $10(b)$ that the dye adsorption to the PDMS wall drastically changes the electroosmotic mobility. In fact, the experimental condition was not exactly the same, because after the uncaging event, the cation $\left(\mathrm{K}^{+}\right)$was produced from the CMNB-caged fluorescein. This may induce the slight change in the electroosmotic mobility.

It is noted from Figure $7(b)$ that the electroosmotic flow velocity seems to scatter slightly with respect to time, which is probably caused by the dye diffusion due to its small molecular weight. The velocity gradient near the glass wall for the rinsed case is estimated to be $8.6(-/ \mathrm{s})$ from Figure 9 and this value is much smaller than that of pressure-driven flow, as described in the next subsection. When the scattered velocity data in Figure $7(b)$ is expressed by the ratio of the r.m.s velocity to the mean velocity, the value is calculated at $6.5 \%$ within $0-120 \mathrm{~ms}$, while $5.8 \%$ within $0-40 \mathrm{~ms}, 6.3 \%$ within $41-80 \mathrm{~ms}$ and $7.5 \%$ within $81-120 \mathrm{~ms}$. This estimation indicates roughly that the dye velocity by EWMT was almost constant, in other words, the uncaged dye illuminated by the evanescent wave remained in the depthwise region, roughly from the wall surface to $175 \mathrm{~nm}$ (this corresponds to 0.1 of $\left.I_{\text {eva }}(0)^{(28)}\right)$.

\subsection{Dye Dispersion in Pressure-Driven Flow}

Measurements of the dye motion in pressure-driven flow were carried out using a glass-PDMS microchannel with a depth of $50 \mu \mathrm{m}$ and a width of $400 \mu \mathrm{m}$ in order to neglect the effect of the spanwise velocity gradient near both the walls on the dye dispersion. The height of water surface in the two reservoirs was controlled to generate pressure-driven flow. One example of the streamwise velocity profiles obtained from MT by volume illumination using $20 \times$ objective lens (Nikon Corp., CIF $\mathrm{S}$ Fluor, $N A=0.75$, a depth-of-field of $3.06 \mu \mathrm{m})$ is shown in Figure 11(a) in comparison with the theoretical result by Brody et al. ${ }^{(16)}$. The Reynolds number based on a channel equivalent diameter of $88.9 \mu \mathrm{m}$ and a bulk velocity of $638 \mu \mathrm{m} / \mathrm{s}$ was $6.4 \times 10^{-2}$. The trapezoidal shape was obtained in contrast to the reports using a capillary or a tube $\mathrm{e}^{(10),(13),(15)}$.

The instantaneous velocities at $y=200 \mu \mathrm{m}$ in time series by EWMT and MT using the CMOS camera were plotted in Figure $11(b)$. The velocity data by EWMT scattered significantly and the estimation used in the previous subsection indicates $46.3 \%$ within 0-180 ms, while $27.2 \%$ within $0-60 \mathrm{~ms}, 33.1 \%$ within $61-120 \mathrm{~ms}$ and $61.2 \%$ within 121-180 ms. This may be caused by the large velocity gradient near the wall $(94.4(-/ \mathrm{s})$ calculated from the theoretical result), however, it is difficult using the experimental results to examine the relationship between the velocity gradient and the dye dispersion in the 
(a)

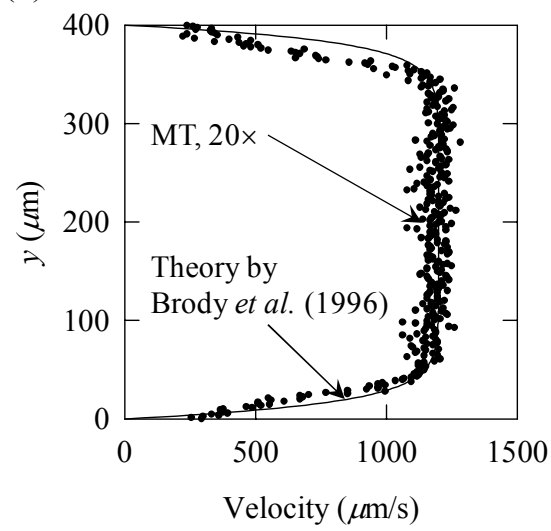

(b)

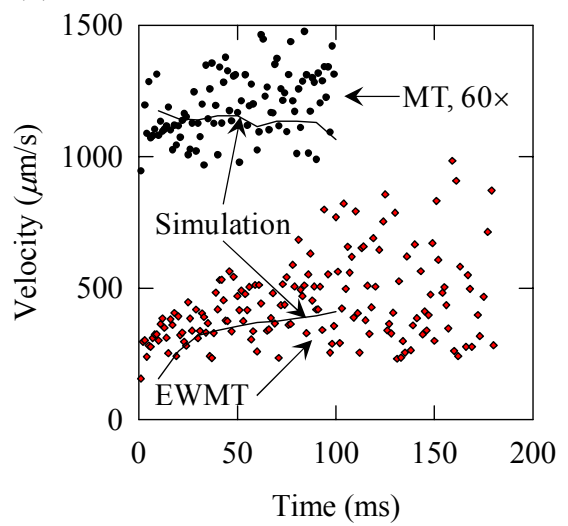

Figure 11. (a) Velocity profile of pressure-driven flow obtained from MT by volume illumination with the $20 \times$ objective lens using the CMOS camera in comparison with the theoretical result by Brody et $a{ }^{(16)}$ and $(b)$ the time evolution of the pressure-driven flow velocity at $y=200 \mu \mathrm{m}$ obtained from EWMT and MT by volume illumination with the $60 \times$ objective lens using the CMOS camera in comparison with the numerical simulations. Measurement was performed just after $74 \mathrm{~ms}$ of the UV laser exposure, thus, for example, 4 ms corresponds to $t=t_{0}+4 \mathrm{~ms}\left(t_{0}=74 \mathrm{~ms}\right)$. The centerline velocity was calculated to be $1.19 \times 10^{3} \mu \mathrm{m} / \mathrm{s}$.

depthwise (Z-) direction. In the present study, the numerical simulations based on the finite volume method were performed to investigate the near-wall motion of dye in pressure-driven flow. The Navier-Stokes and the scalar equations were solved using properties of water. The fluid flow was calculated with the periodic boundary condition. The dye velocity was obtained by the displacement between the peak values of the successive concentration profiles divided by the time step of $10 \mathrm{~ms}$.

The two-dimensional simulations were first performed using a control volume of $(\Delta x$, $\Delta z)=(0.1 \mu \mathrm{m}, 0.1 \mu \mathrm{m})$ and $(0.2 \mu \mathrm{m}, 0.2 \mu \mathrm{m})$ in the computational domain of $x=0-200 \mu \mathrm{m}$ and $z=0-50 \mu \mathrm{m}$. Two types of mesh were utilized to validate the simulation technique. In this subsection, the results using $(\Delta x, \Delta z)=(0.1 \mu \mathrm{m}, 0.1 \mu \mathrm{m})$ are presented. Initially the dye with a diffusivity of $5.2 \times 10^{-10} \mathrm{~m}^{2} / \mathrm{s}$, which was the mean value of dye diffusivity by EWMT and MT listed in Table 2, was located $x=50 \mu \mathrm{m}$ with a Gaussian function (the standard deviation of 0.5 ) within $0-10 \mathrm{~ms}$. The dye velocities at $z=76 \mathrm{~nm}$ (the characteristic penetration depth) and $z=25 \mu \mathrm{m}$ (the depthwise midpoint) are shown in Figure $11(b)$. The dye motion at $z=25 \mu \mathrm{m}$ seemed to be almost constant, while the dye velocity at $z=76 \mathrm{~nm}$ increased with respect to time. This suggests that the dye convected to the streamwise $(X-)$ direction disperses toward the glass wall, which may be induced by the concentration gradient of the dye.

Figure 12 shows the time evolution of the pressure-driven flow velocity at the Reynolds number of $9.0 \times 10^{-3}$ measured by EWMT and MT using the CCD camera. The similar trend was observed. The three-dimensional simulation was conducted using a control volume of $\Delta x=2 \mu \mathrm{m}, \Delta y=40 \mu \mathrm{m}, \Delta z=0.5 \mu \mathrm{m}$ in the computational domain of $x=0-200 \mu \mathrm{m}, y=$ $0-400 \mu \mathrm{m}$ and $z=0-50 \mu \mathrm{m}$. The dye with a diffusivity of $5.2 \times 10^{-10} \mathrm{~m}^{2} / \mathrm{s}$ was located $x=50$ $\mu \mathrm{m}$ within $0-10 \mathrm{~ms}$. The dye concentration existed in the depthwise region from $z=0$ to $z=$ $500 \mathrm{~nm}$ was integrated and then the dye velocity was calculated using the peak values of the integrated concentration, as shown in Figure 12. The simulation result was also increased with respect to time and showed the same tendency as Figure 11(b). It is surmised from these results that the dye disperses from the channel center to the wall due to the concentration gradient that is generated by the fluid convection. The simulation results in Figures $11(b)$ and 12 were fitted with the following equation 


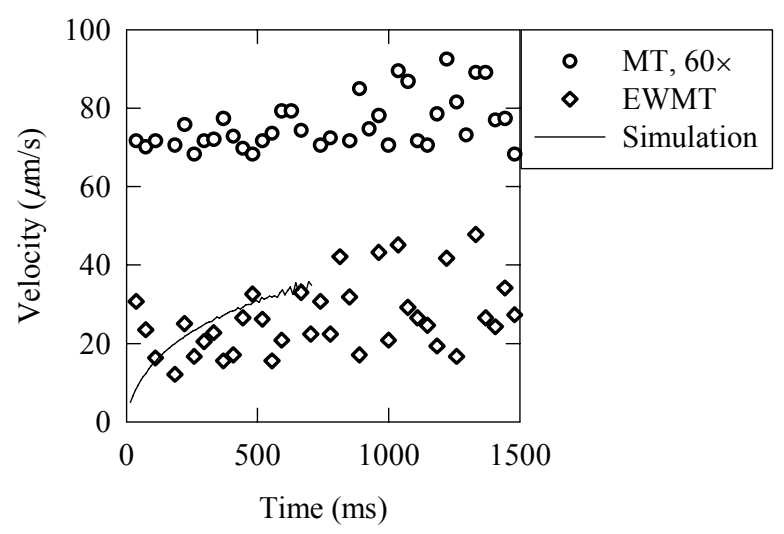

Figure 12. The time evolution of the pressure-driven flow velocity at $y=200 \mu \mathrm{m}$ obtained from MT by volume illumination with the $60 \times$ objective lens and EWMT using the CCD camera in comparison with the numerical simulation. Measurement was performed just after $74 \mathrm{~ms}$ of the UV laser exposure, thus, for example, $74 \mathrm{~ms}$ corresponds to $t=t_{0}+74 \mathrm{~ms}$ $\left(t_{0}=74 \mathrm{~ms}\right)$. The centerline velocity was calculated to be $81 \mu \mathrm{m} / \mathrm{s}$.

$$
U(t)=\alpha \sqrt{t}+U(0),
$$

where $U$ is the dye velocity, $t$ is time and $\alpha$ is the coefficient which represents that the dye dispersion near the wall by the fluid convection is similar to the development of the boundary layer ${ }^{(29)}$. The dye velocities at $t=0$ in Figures $11(b)$ and 12 are calculated to be 56 $\mu \mathrm{m} / \mathrm{s}$ and $2.6 \mu \mathrm{m} / \mathrm{s}$, respectively. The locations of these velocities are estimated at $z \approx 590$ $\mathrm{nm}$ and $z \approx 510 \mathrm{~nm}$ by using the theoretical velocity profiles, which was calculated by Brody et al. ${ }^{(16)}$. It is noted from Figures 11 and 12 that the dye dispersion toward the wall was significant with respect to time especially in the vicinity of the wall.

\section{Conclusions}

A new molecular tagging technique was developed to investigate the direct influence of the dye on elelctroosmotic flow and the dye dispersion near the microchannel wall in pressure-driven flow. The evanescent wave was utilized to illuminate the uncaged dye existed near the glass wall. The experimental results were compared with those by micro-PIV and the numerical simulations. The important conclusions obtained from this work are summarized below.

(1) In comparison with the near wall measurements by micro-PIV with a confocal scanner, the adsorption of the caged fluorescent dye to the microchannel wall changed drastically the elelctroosmotic mobility (the zeta-potential) of the wall, which can be directly measured by EWMT.

(2) The velocity of the uncaged dye in pressure-driven flow measured by EWMT increased with respect to time, which was not observed in the experiments of MT by volume illumination and of electroosmotic flow. The large velocity gradient near the wall created the concentration gradient of the dye, which induced the dye dispersion toward the wall.

Further development of EWMT will contribute to investigate the interactions between the nanoscopic objects and the electrolyte-solid interface.

\section{Acknowledgements}

This work was subsidized by Grant-in-Aid for Young Scientists of Ministry of Education, Culture, Sports, Science and Technology in Japan (No. 17686017) and TEPCO 
Research Foundation.

\section{References}

(1) Reyes, D.R., Iossifidis, D., Auroux, P.A. and Manz, A., Anal. Chem., Vol. 74, (2002), pp. 2623-2636.

(2) Auroux, P.A., Iossifidis, D., Reyes, D.R. and Manz, A., Anal. Chem., Vol. 74, (2002), pp. 2637-2652.

(3) Sinton, D.A., (2004) Microfluidics Nanofluidics, Vol. 1, (2004), pp. 2-21.

(4) Shinohara, K., Sugii, Y., Aota, A., Hibara, A., Tokeshi, M., Kitamori, T. and Okamoto, K., Meas. Sci Technol., Vol. 15, (2004), pp. 1965-1970.

(5) Sato, Y., Inaba, S., Hishida, K. and Maeda, M., Exp. Fluids, Vol. 35, (2003), pp. 167-177.

(6) Kihm, K.D., Banerjee, A., Choi, C.K. and Takagi, T., Exp. Fluids, Vol. 37, (2004), pp. 811-824.

(7) Sadr, R., Yoda, M., Zheng, Z. and Conlisk, A.T., J. Fluid Mech., Vol. 506, (2004), pp. 357-367.

(8) Gendrich, C.P. and Koochesfahani, M.M., Exp. Fluids, Vol. 22, (1996), pp. 67-77.

(9) Koochesfahani, M.M., Cohn, R. and MacKinnon, C., Meas. Sci. Technol., Vol. 11, (2000), pp. 1289-1300.

(10) Paul, P.H., Garguilo, M.G. and Rakestraw, D.J., Anal. Chem., Vol. 70, (1998), pp. 2459-2467.

(11) Herr, A.E., Molho, J.I., Santiago, J.G., Mungal, M.G., Kenny, T.W. and Garguilo, M.G., Anal. Chem., Vol. 72, (2000), pp. 1053-1057.

(12) Ross, D., Johnson, T.J. and Locascio, L.E., Anal. Chem., Vol. 73, (2001), pp. 2509-2515

(13) Maynes, D. and Webb, A.R., Exp. Fluids, Vol. 32, (2002), pp. 3-15.

(14) Sinton, D.A, Escobedo-Canseco, C., Ren, L. and Li, D., J. Colloid. Interface Sci., Vol. 254, (2002), pp. 184-189.

(15) Sinton, D.A. and Li, D., Int. J. Therm. Sci., Vol. 42, (2003), pp. 847-855.

(16) Brody, J.P., Yager, P., Goldstein, R.E. and Austin, R.H., Biophysical. J., Vol. 71, (1996), pp. 3430-3441.

(17) Ross, D. and Locascio, L.E., Anal. Chem., Vol. 75, (2003), pp. 1218-1220.

(18) Pappaert, K., Biesemans, J., Clicq, D., Vankrunkelsven, S. and Desmet, G., Lab Chip, Vol. 5, (2005), pp. 1104-1110.

(19) Lempert, W.R., Magee, K., Ronney, P., Gee, K.R. and Haugland, R.P., Exp. Fluids, Vol. 18, (1995), Vol. 249-257.

(20) Kihm, K.D., Banerjee, A., Choi, C.K. and Takagi, T., Exp. Fluids, Vol. 37, (2004), pp. 811-824.

(21) Hain, R., Kähler, C.J. and Tropea, C., Exp. Fluids, Vol. 42, (2007), pp. 403-411.

(22) Inoue, S. and Spring, K.R., Video Microscopy, the fundamentals, 2nd edition, (1997), Plenum.

(23) Probstein, R.F., Physicochemical hydrodynamics, An introduction second edition, (1994), John Willy \& Sons.

(24) Galambos, P. and Forster, F.K., Micro Total Analysis Systems '98, (1998), pp. 189-191.

(25) Huang, X., Gordon, M.J. and Zare, R.N., Anal. Chem., Vol. 60, (1998), pp. 1837-1838.

(26) Park, J.S., Choi, C.K. and Kihm, K.D., Exp. Fluids, Vol. 37, (2004), pp. 105-119.

(27) Ichiyanagi, M., Sato, Y. and Hishida, K., Exp. Fluids, Vol. 43, (2007), pp. 425-435.

(28) Zettner, C.M. and Yoda, M., Exp. Fluids, Vol. 34, (2003), pp. 115-121.

(29) Schlichting, H., Boundary-Layer Theory, (1979), McGraw-Hill, Inc. 